\title{
Colour implications of self-association processes of wine anthocyanins
}

\author{
Susana González-Manzano • Celestino Santos-Buelga • \\ Montserrat Dueñas · Julián C. Rivas-Gonzalo · \\ Teresa Escribano-Bailón
}

Received: 27 July 2006/ Revised: 17 December 2006/ Accepted: 8 January 2007 / Published online: 9 February 2007

(C) Springer-Verlag 2007

\begin{abstract}
Copigmentation processes have been indicated to be crucial to stabilise coloured forms of the anthocyanins and explain colour expression in young red wines. Several studies exist about copigmentation between anthocyanins and different phenolics in model solutions, but little information is available about interactions among anthocyanins themselves. In this work, the process of self-association has been investigated in wine-like model solutions containing different grape anthocyanins (the 3-glucosides of malvidin, delphinidin and peonidin). The results obtained confirmed the existence of anthocyanin self-association and its influence on the apparent hydration constant of the anthocyanins with subsequent modification in the colour of the solutions. It was observed that the greater the degree of methoxylation of the anthocyanin B-ring the greater was the magnitude of the self-association. Colour analyses in the CIELAB space showed that self-association produces changes, which are more important in quantitative parameters (chroma, $C_{\mathrm{ab}}^{*}$ and lightness, $L^{*}$ ) than in qualitative ones (hue, $h_{\mathrm{ab}}$ ). Selfassociation leads to an increase in $C_{\mathrm{ab}}^{*}$, indicating a more intense colour of the solutions, and to a decrease in the psychometric index $L^{*}$, meaning that a darkening is produced. The effects on the colour were more pronounced with the passage of time of storage of the solutions.
\end{abstract}

S. González-Manzano · C. Santos-Buelga ·

M. Dueñas - J. C. Rivas-Gonzalo .

T. Escribano-Bailón $(\bowtie)$

Grupo de Investigación en Polifenoles,

Unidad de Nutrición y Bromatología, Facultad de Farmacia,

Universidad de Salamanca, Campus Miguel de Unamuno,

37007 Salamanca, Spain

e-mail: escriban@usal.es
Keywords Self-association · Copigmentation · Wine $\cdot$ Anthocyanins $\cdot$ Colour

\section{Introduction}

The anthocyanins are plant pigments responsible for the colour of many flowers and fruits, as well as processed products derived from these latter, such as jams, juices or red wine. In aqueous solution they exist, basically, in the form of four species in equilibrium depending on the $\mathrm{pH}$ : quinonoidal base, flavylium cation, hemiketal and chalcone. The distribution among these forms depends on the $\mathrm{pH}$, temperature, composition of the medium and the molecular interactions that may exist among anthocyanins and other compounds. The constants of hydration $\left(K_{\mathrm{h}}\right)$ and proton-transfer $\left(K_{\mathrm{a}}\right)$ are thermodynamic constants, which allow the definition of the distribution among anthocyanin forms in acidic to neutral aqueous solutions. In a weakly acid $\mathrm{pH}$, such as that which exists in vacuolar media and many processed products, the structure that should predominate is the colourless hydrated hemiketal form. Nevertheless, the molecules corresponding to the coloured forms (flavylium and quinonoidal base) of the anthocyanins can interact in a non-covalent way among themselves or with other compounds. These interactions provoke displacements of the anthocyanin equilibria towards the coloured structures and, in this way, limit the decolouring effect of the low acidity. This process, named copigmentation, is known to contribute to the expression of the colour of the anthocyanins in flowers and fruits. The copigmentation complexes are easily disrupted by dilution, allowing the anthocyanins to return to their expected equilibria 
between structural forms depending on the $\mathrm{pH}$. This feature can be used to distinguish the colour due to copigmentation [1].

In the literature there are several studies (see e.g., [2-5]) of intermolecular copigmentation among anthocyanins and other compounds, generally phenolics, but there is less information about the interactions that can take place among the anthocyanins themselves. The self-association of the anthocyanins was first suggested by Asen et al. [2] and is demonstrated as a positive deviation from Beer's law, which occurs on increasing the concentration of the anthocyanin. The complexes of self-association are formed by vertical stacking of the molecules of anthocyanins and are stabilised principally through the hydrophobic interactions that take place between their aromatic nuclei [6-10]. The effect can be compared to that of copigmentation among anthocyanins and other phenolic compounds, although on the contrary to this, the self-association might produce a hypsochromic shift in the wavelength of maximum absorption $[8,9]$. It has been indicated that for the self-aggregation of the anthocyanins to take place, they should be found in the solutions at concentrations greater than $1 \mathrm{mmol} / \mathrm{L}$ $[2,8,9,11-15]$. Thus, although it is accepted that the phenomenon could be involved in the colour of flowers [9, 16], in wine its participation would be arguable, given the relatively low concentration of anthocyanins. In addition, the existence of ethanol in the medium is a factor which would further limit the interaction, since the presence of organic solvents weakens the intermolecular hydrophobic interactions, provoking the destabilisation of the complexes formed [12, 17].

In this work the existence of processes of self-association is demonstrated in wine-like solutions $(12 \%$ ethanol, $\mathrm{pH}$ 3.6) which contain anthocyanins and ethanol in concentrations similar to those habitually found in red wines. The influences of the self-association on the hydration apparent constant $\left(K=K_{\mathrm{h}}+K_{\mathrm{a}}\right)$, as well as on the colour of the solutions and the stability of the anthocyanins are also studied.

\section{Materials and methods}

Reagents and standards

All organic solvents were HPLC grade and were purchased from Merck, Darmstadt, Germany. Gallic acid, vanillic acid and syringic acid were provided by SigmaAldrich Chemie, Steinheim, Germany.
Preparation of anthocyanins

Anthocyanins [malvidin 3-glucoside (Mv3g), peonidin 3-glucoside (Pn3g), and delphinidin 3-glucoside $(\mathrm{Dp} 3 \mathrm{~g})]$ were isolated from grape skins by semi-preparative HPLC as described in Heredia et al. [18].

\section{Model solutions}

Solutions containing individual anthocyanins (Mv3g, Pn3g or Dp3g) were prepared in the range of 50 $600 \mathrm{mg} / \mathrm{L}$ (ca. $0.1-1.2 \mathrm{mmol} / \mathrm{L})$ in $12 \%$ ethanol $(v / v)$ containing $5 \mathrm{~g} / \mathrm{L}$ of tartaric acid buffered with $1 \mathrm{~mol} / \mathrm{L}$ $\mathrm{NaOH}$ to $\mathrm{pH} 3.6$ (wine-like solution). The solutions were stored in darkness at $25{ }^{\circ} \mathrm{C}$ for 4 weeks. All solutions were prepared in triplicate.

\section{Spectra measurements}

Absorption spectra of the solutions were recorded in the visible range from 380 to $770 \mathrm{~nm}$ in a Hewlett Packard UV-vis HP3853 spectrophotometer in a $10 \mathrm{~mm}$ pathlength quartz cell thermostatted at $25^{\circ} \mathrm{C}$. Spectra were also obtained after diluting the solutions twentyfold with the wine-like solution. The magnitude of the hyperchromic effect due to the self-association process was estimated in each solution by measuring the relative increase of the absorbance at $\lambda_{\text {max }}:\left(A_{\mathrm{c}}-A_{\mathrm{d}} / A_{\mathrm{c}}\right) . A_{\mathrm{c}}$ being the absorbance of the solution at $\lambda_{\max }$ and $A_{\mathrm{d}}$ the value obtained after multiplying by the dilution factor, the absorbance at $\lambda_{\max }$ of the solutions diluted 1:20 with the $\mathrm{pH} 3.6$ wine-like solution [1].

\section{Calculation of $K$ values}

This was carried out as described by Dueñas et al. [19]. Solutions at different $\mathrm{pH}$ values (range 0.9-4.2) were prepared for each anthocyanin (Mv3g, Pn3g and Dp3g) at a concentration of $2 \times 10^{-2} \mathrm{mmol} / \mathrm{L}$. Ionic strength was adjusted to $1 \mathrm{~mol} / \mathrm{L}$ by $\mathrm{NaCl}$. The solutions were kept for $2 \mathrm{~h}$ at $25^{\circ} \mathrm{C}$ to ensure complete hydration equilibrium and their UV-vis spectra recorded. The value of first-order apparent rate constant of the hydration reaction $K\left(K=K_{\mathrm{h}}+K_{\mathrm{a}}\right)$ was obtained from the Eq. 1 described by Dangles et al. [20]

$\frac{A_{0}}{A_{0}-A}=\frac{K_{\mathrm{h}}+K_{\mathrm{a}}}{K_{\mathrm{h}}+K_{\mathrm{a}}\left(1-r_{\mathrm{A}}\right)}+\frac{10^{-\mathrm{pH}}}{K_{\mathrm{h}}+K_{\mathrm{a}}\left(1-r_{\mathrm{A}}\right)}$

In this equation, $A_{0}$ is the absorbance of a strongly acidic solution $(\mathrm{pH}<1)$ in which the anthocyanin is in pure flavylium form and $A$ is the absorbance at a given 
slightly acidic $\mathrm{pH}$. Parameter $r_{\mathrm{A}}$ is the ratio of molar absorption coefficients of the same pigment in its quinonoidal form and in its flavylium form.

The $K$ values of the solutions containing increasing anthocyanin concentrations $(0.1-1.2 \mathrm{mmol} / \mathrm{L})$ were calculated by substituting the values of absorbance at $\mathrm{pH}<1\left(A_{0}\right)$ and at $\mathrm{pH} 3.6(A)$ in the same formula.

\section{HPLC analyses}

These were performed in a Hewlett-Packard 1100 equipment (Agilent Technologies, Waldbronn, Germany) provided with a quaternary pump, automatic injector, diode array detector and data treatment station using the method described by Alcalde-Eon et al. [21]. Separation was achieved on a $3 \mu \mathrm{m}$ Spherisorb C-18 $150 \times 4.6 \mathrm{~mm}$ column (Waters) thermostatted at $35{ }^{\circ} \mathrm{C}$. The solvents used were (A) $0.1 \%$ trifluoroacetic acid (TFA) in water, and (B) gradientgrade acetonitrile, establishing the following gradient: $10-15 \%$ B for $5 \mathrm{~min}$, isocratic $15 \%$ B for $5 \mathrm{~min}, 15-$ $18 \% \mathrm{~B}$ for $5 \mathrm{~min}, 18-35 \% \mathrm{~B}$ for $25 \mathrm{~min}$ and $35-50 \%$ $\mathrm{B}$ for $5 \mathrm{~min}$, followed by washing and reconditioning of the column. The flow rate was $0.5 \mathrm{~mL} / \mathrm{min}$. Detection was carried out in a photodiode spectrophotometer, using 280 and $520 \mathrm{~nm}$ as the preferred wavelengths.

\section{Tristimulus colorimetry}

The whole visible spectrum $(380-770 \mathrm{~nm})$ of each sample was recorded $(\Delta \lambda=1 \mathrm{~nm})$. The CIE 1964 standard observer ( $10^{\circ}$ visual field) and the CIE standard illuminant $\mathrm{D}_{65}$ were used as references. The CIELAB parameters $\left(L^{*}, a^{*}, b^{*}, C_{\mathrm{ab}}^{*}, h_{\mathrm{ab}}\right)$ were determined by using the original CromaLab ${ }^{\circledR}$ software [22], following the recommendations of the Commission Internationale de L'Eclairage [23]. Parameters were calculated for both the original non-diluted solutions and for the same solutions diluted 20-fold after multiplying the absorbance values by the dilution factor.

Colour differences $\left(\Delta E_{\mathrm{ab}}^{*}\right)$ between the solution with self-association and without self-association were calculated with the equation

$\Delta E_{\mathrm{ab}}^{*}=\left[\left(\Delta L^{*}\right)^{2}+\left(\Delta a^{*}\right)^{2}+\left(\Delta b^{*}\right)^{2}\right]^{1 / 2}$

where $\Delta L^{*}, \Delta a^{*}$ and $\Delta b^{*}$ were the differences between the CIELAB parameters calculated from the original non-diluted solution and after multiplying values of the absorbance of the same solution diluted 20 -fold by the dilution factor.

\section{Results and discussion}

Figure 1 shows the absorption spectra of the solutions containing increasing concentrations of Mv3g (0.1$1.2 \mathrm{mmol} / \mathrm{L}$ ) and those obtained after multiplying the values of the absorbance of the same solutions diluted 1:20 by 20 . The dilution was expected to dissociate the possible complexes of self-association in the solutions [1]. As expected, an increase was produced in the absorbance values in the visible region with the increase in the anthocyanin concentration in the solution. However, the magnitude of this increase was greater in the non-diluted solutions, indicating that a deviation from Beer's law occurs and, therefore, the existence of a possible self-association process. Similar behaviour was found for the solutions of Pn3g and Dp3g.

In the most diluted solutions prepared (concentration of anthocyanin $2 \times 10^{-2} \mathrm{mmol} / \mathrm{L}$ ) an almost complete disappearance of the visible band, without bathochromic effect, was observed with the increase of the $\mathrm{pH}$ from 0.9 to 4.2 . This indicates that the anthocyanin undergoes pure hydration in the $\mathrm{pH}$ range investigated [19] and, therefore, its $\mathrm{p} K$ value must be essentially equal to $\mathrm{p} K_{\mathrm{h}}$. The $\mathrm{p} K$ values obtained in these conditions were 2.8 for $\mathrm{Mv} 3 \mathrm{~g}, 2.6$ for Dp3g and
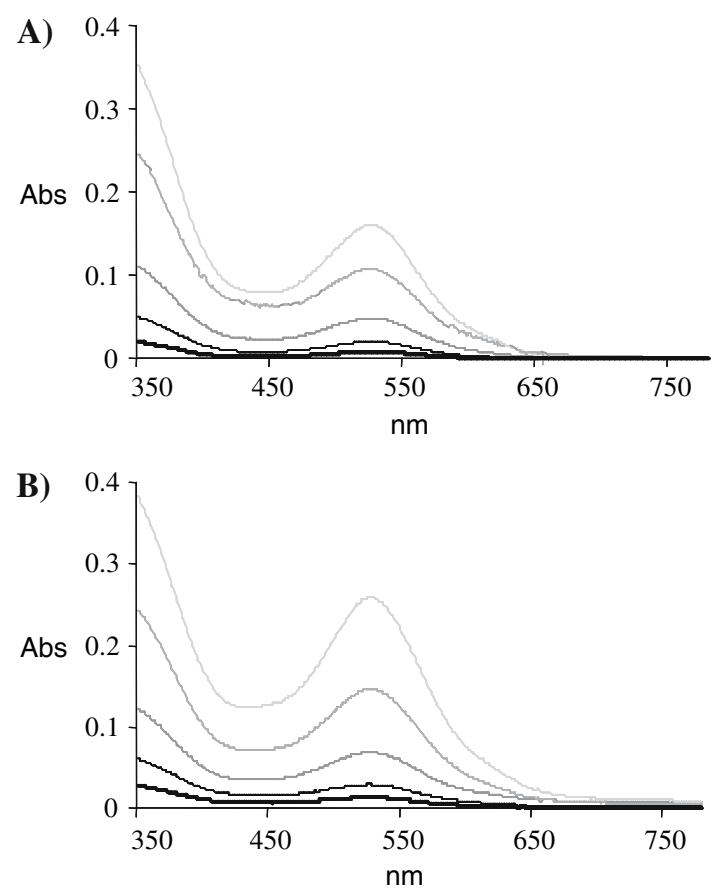

Fig. 1 a spectra obtained with the values resulting after multiplying the values of the absorbance solutions after dilution 1:20 with wine-like solution $(\mathrm{pH} 3.6)$ by 20 (dilution factor). b Spectra of absorption of the non-diluted solutions of Mv3g. Concentrations were $0.1,0.2,0.4,0.8$ and $1.2 \mathrm{mmol} / \mathrm{L}$ (lines from darker to clearer on increasing the concentration) 
2.9 for Pn3g. The value for Mv3g is in accordance with that reported by Dueñas et al. [19] and that of Dp3g is similar to that found by Figueiredo et al. [24] $\left(\mathrm{p} K_{\mathrm{h}}=2.4\right)$. The slight difference observed may be due to the different ionic strength used in each case $(1 \mathrm{~mol} / \mathrm{L}$ adjusted by $\mathrm{NaCl}$ in our case, and $0.2 \mathrm{~mol} / \mathrm{L}$ by Figueiredo et al.). According to our knowledge, this is the first time that the $\mathrm{p} K$ value for Pn3g has been obtained.

In order to confirm the existence of self-association in the solutions with increasing concentrations of the anthocyanins, the apparent hydration constants $\left(\mathrm{p} K=\mathrm{p} K_{\mathrm{h}}+\mathrm{p} K_{\mathrm{a}}\right)$ were calculated. An increase in the $\mathrm{p} K$ values in relation to the one existing in the most diluted solution would indicate an increasing stability of the anthocyanin towards hydration, which should be expected if self-association occurs. The results shown in Table 1 clearly demonstrate that an increase in the $\mathrm{p} K$ values is produced as the concentration of the anthocyanin increases in the solutions. Since no other compounds able to interact with the anthocyanins are present in the solutions, this can only be explained as a result of the self-association process.

Figure 2 shows the molar fractions of the coloured forms $\left(\mathrm{AH}^{+}+\mathrm{A}\right)$ for the three studied anthocyanins in the solutions containing different concentrations calculated from their $\mathrm{p} K$ values. It can be observed that the percentage of coloured forms increases with the increase in the concentration of anthocyanin in the solutions. The magnitude of the increase in the $\mathrm{p} K$ values and subsequently the percentage of coloured forms in the solutions vary with the anthocyanin involved. It is greater for Mv3g followed by Pn3g and Dp3g, suggesting that the effectiveness of self-association improves when the number of methoxyl groups in the $\mathrm{B}$ ring increases.

Table $1 \mathrm{p} K$ values obtained increasing the anthocyanin concentration in the wine like solutions

\begin{tabular}{llll}
\hline $\begin{array}{l}\text { Concentration } \\
(\mathrm{mmol} / \mathrm{L})\end{array}$ & $\mathrm{p} K \mathrm{Mv3g}$ & $\mathrm{p} K \mathrm{Pn} 3 \mathrm{~g}$ & $\mathrm{p} K \mathrm{Dp} 3 \mathrm{~g}$ \\
\hline $2 \times 10^{-2}$ & 2.80 & 2.92 & 2.60 \\
0.1 & 2.90 & 3.31 & 2.55 \\
0.2 & 3.00 & 3.33 & 2.71 \\
0.3 & 3.06 & 3.37 & 2.74 \\
0.4 & 3.13 & 3.43 & 2.83 \\
0.5 & 3.21 & 3.51 & 2.89 \\
0.6 & 3.28 & 3.54 & 2.87 \\
0.7 & 3.39 & 3.55 & 2.95 \\
0.8 & 3.41 & 3.60 & 2.97 \\
0.9 & 3.45 & 3.60 & 3.01 \\
1 & 3.48 & 3.65 & 3.05 \\
1.1 & 3.51 & 3.68 & 3.07 \\
1.2 & 3.52 & 3.70 & 3.08 \\
\hline
\end{tabular}

The solutions of the three anthocyanins in the concentration range from 0.1 to $1.2 \mathrm{mmol} / \mathrm{L}$ were kept in darkness at $25{ }^{\circ} \mathrm{C}$ for 28 days. With the passage of time the absorbance in the visible region of the solutions exhibited a progressive increase in the whole range of concentrations studied, although with different magnitudes depending on the anthocyanin and the concentration. This can be seen in Fig. 3, where the relative increase of the absorbance at $\lambda_{\text {max }}:\left(A_{\mathrm{c}}-A_{\mathrm{d}} / A_{\mathrm{c}}\right)$ against time is represented. This increase could not be attributable to an effect of evaporation of the solvent, since the vials remained hermetically closed and no significant decrease in the volume of the solutions was appreciated. On the contrary, as will be discussed later, there was a certain degradation of the anthocyanins towards colourless products, which made the hyperchromic displacement even more difficult to explain. It can, therefore, be supposed that the formation of the complexes of self-association is not an immediate process, but the interactions among molecules are established progressively and the more slowly the more diluted the solution is. In support of the progressive establishment of the complexes of self-association was the observation of the slight hypsochromic displacement (between 4 and $6 \mathrm{~nm}$ ) produced with time in the wavelength of maximum absorbance in the visible region of the solutions, an aspect associated by some authors to the phenomenon of self-association [8].

During the storage of the solutions a decrease in the concentration of the anthocyanins was produced (Fig. 4), indicating that the existence of self-association does not impede their degradation. Dp3g proved to be the most unstable anthocyanin among those studied. This can be related to the trihydroxyl system in its B ring, as has been suggested elsewhere [25]. The decrease in anthocyanins was accompanied by the formation of colourless products (Fig. 5), among which syringic, vanillic and gallic acids could be identified in the solutions of Mv3g, Pn3g and Dp3g, respectively. Their identification was made from their UV spectra and retention times compared with authentic standards. These phenolic acids would result from the fragments corresponding to the $\mathrm{B}$ ring released after hydrolytic cleavage of each anthocyanin [26]. It might be considered that the phenolic acids formed could act as possible copigments of the anthocyanins, being responsible in part for the hyperchromic effect observed in the solutions. Nonetheless, the low molar phenolic acid/anthocyanin ratio reached could not justify the existence of an effect of copigmentation in the cases of the Mv3g and Pn3g solutions (approximately $1: 30$ at the end of 4 weeks of storage in the more concentrated anthocyanin solutions) (Table 2). 

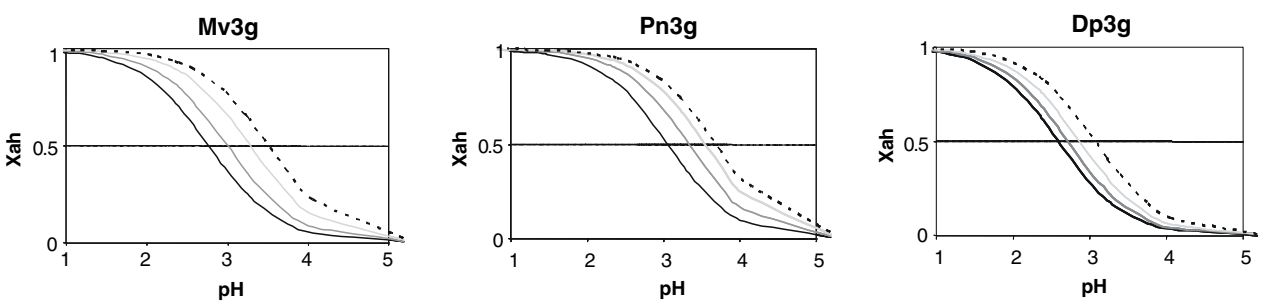

Fig. 2 Molar fractions of $\mathrm{AH}^{+}+\mathrm{A}(\mathrm{Xah})$ in solutions containing increasing concentrations of anthocyanin (Mv3g, Pn3g and Dp3g), thick solid line $2 \times 10^{-2} \mathrm{mmol} / \mathrm{L}$, thin solid line $0.2 \mathrm{mmol} / \mathrm{L}$, thin dashed line $0.6 \mathrm{mmol} / \mathrm{L}$, thick dashed line $1.2 \mathrm{mmol} / \mathrm{L}$
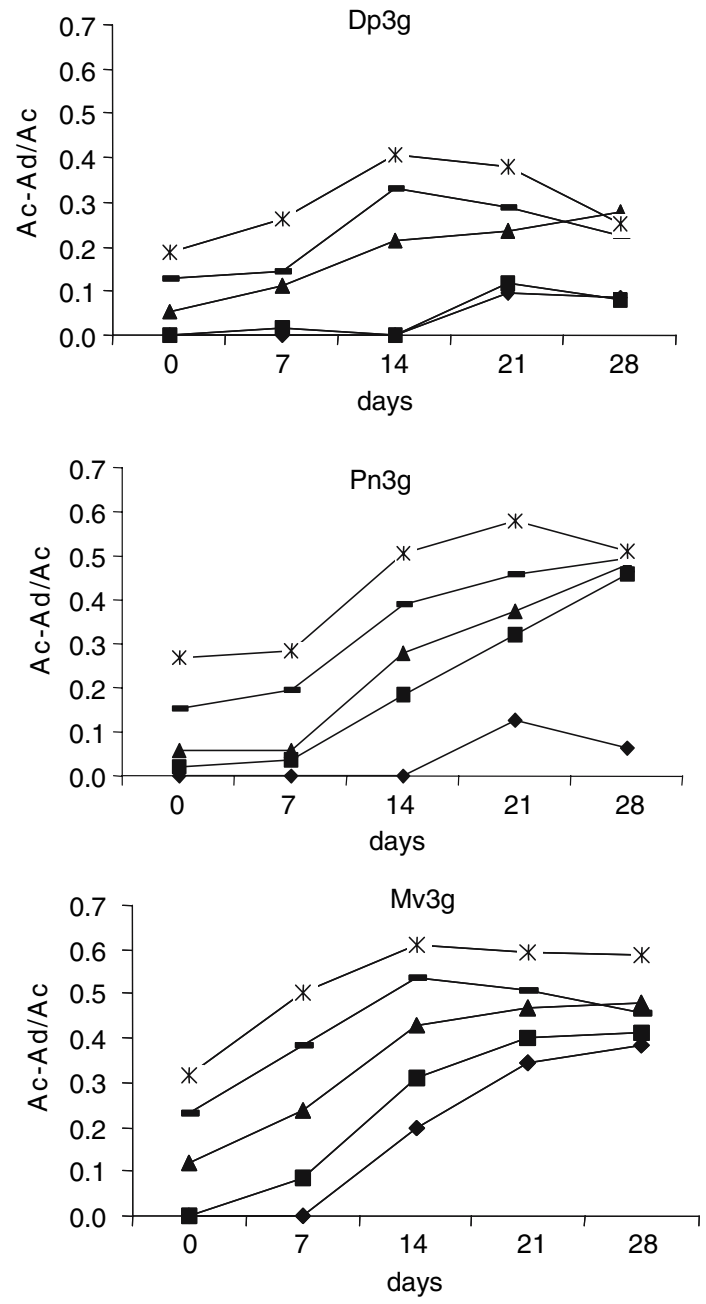

Fig. 3 Relative increase of the absorbance at $520 \mathrm{~nm}\left(A_{\mathrm{c}}-A_{\mathrm{d}} /\right.$ $A_{\mathrm{c}}$ ) in the solutions of $0.1 \mathrm{mmol} / \mathrm{L}$ (filled diamond), $0.2 \mathrm{mmol} / \mathrm{L}$ (filled square), $0.4 \mathrm{mmol} / \mathrm{L}$ (filled triangle), $0.8 \mathrm{mmol} / \mathrm{L}$ (filled rectangle) and $1.2 \mathrm{mmol} / \mathrm{L} \mathrm{(*)} \mathrm{of} \mathrm{Mv3g} \mathrm{Pn3g} \mathrm{and} \mathrm{Dp3g} \mathrm{during}$ the 28 days of study

A considerably higher phenolic acid/anthocyanin molar ratio was reached in the Dp3g solution (approximately $3: 1$ at the end of the assay), however, it would be improbable that it could cause an appreciable effect of copigmentation, given the still low molar copigment/

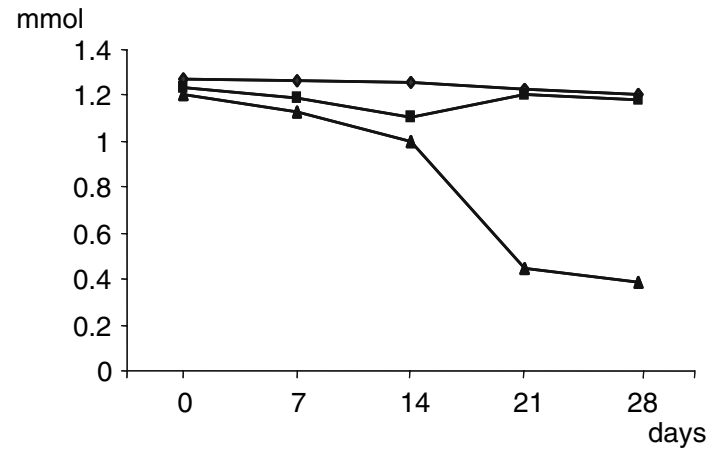

Fig. 4 Evolution of the concentration (mmol) of Mv3g (filled diamond), Pn3g (filled square) and Dp3g (filled triangle) in the solutions containing $1.2 \mathrm{mmol} / \mathrm{L}$ during the 28 days of study

pigment ratio and the fact that gallic acid is considered a bad anthocyanin copigment $[27,28]$.

Colour analysis

With the objective of evaluating the effect of selfassociation in the colour of the solutions, a colour analysis was performed in the CIELAB space. Selfassociation provokes changes in CIELAB parameters that are more important in quantitative parameters (chroma, $C_{\mathrm{ab}}^{*}$ and lightness, $L^{*}$ ) than in qualitative ones (hue, $h_{\mathrm{ab}}$ ) (Table 3). Self-association leads to an increase in the $C_{\mathrm{ab}}^{*}$ values, indicating a more intense red colour of the solutions. The greatest increase was observed in the solutions that contained the anthocyanins with methoxyl groups in the B ring (Mv3g > Pn3g). In some of the solutions of Mv3g and Pn3g with concentration of $1.2 \mathrm{mmol} / \mathrm{L}$ (days 0,14 and $28 \mathrm{in} \mathrm{Mv} 3 \mathrm{~g}$ solutions and days 14 and 28 in Pn3g solutions), the values of the Chroma were lower than in the corresponding solutions where self-association does not exist. This behaviour could be due to the low values of $L^{*}$ (see Table 3) which show that these solutions are very dark and therefore have low quantity of colour (low $C_{\mathrm{ab}}$ * values). Regarding psychometric index of lightness, $L^{*}$, the greater percentage of the coloured forms 
Fig. 5 Chromatograms recorded at $280 \mathrm{~nm}$ of Dp3g,

Pn3g and Mv3g (above, middle and below,

respectively) after 28 days of storage at $25^{\circ} \mathrm{C}$ in the dark. Concentration $1.2 \mathrm{mmol} / \mathrm{L}$.

Peak $1 \mathrm{Dp} 3 \mathrm{~g}$, peak 2 gallic acid, peak 3 Pn3g, peak 4 vanillic acid, peak $5 \mathrm{Mv} 3 \mathrm{~g}$, peak 6 syringic acid
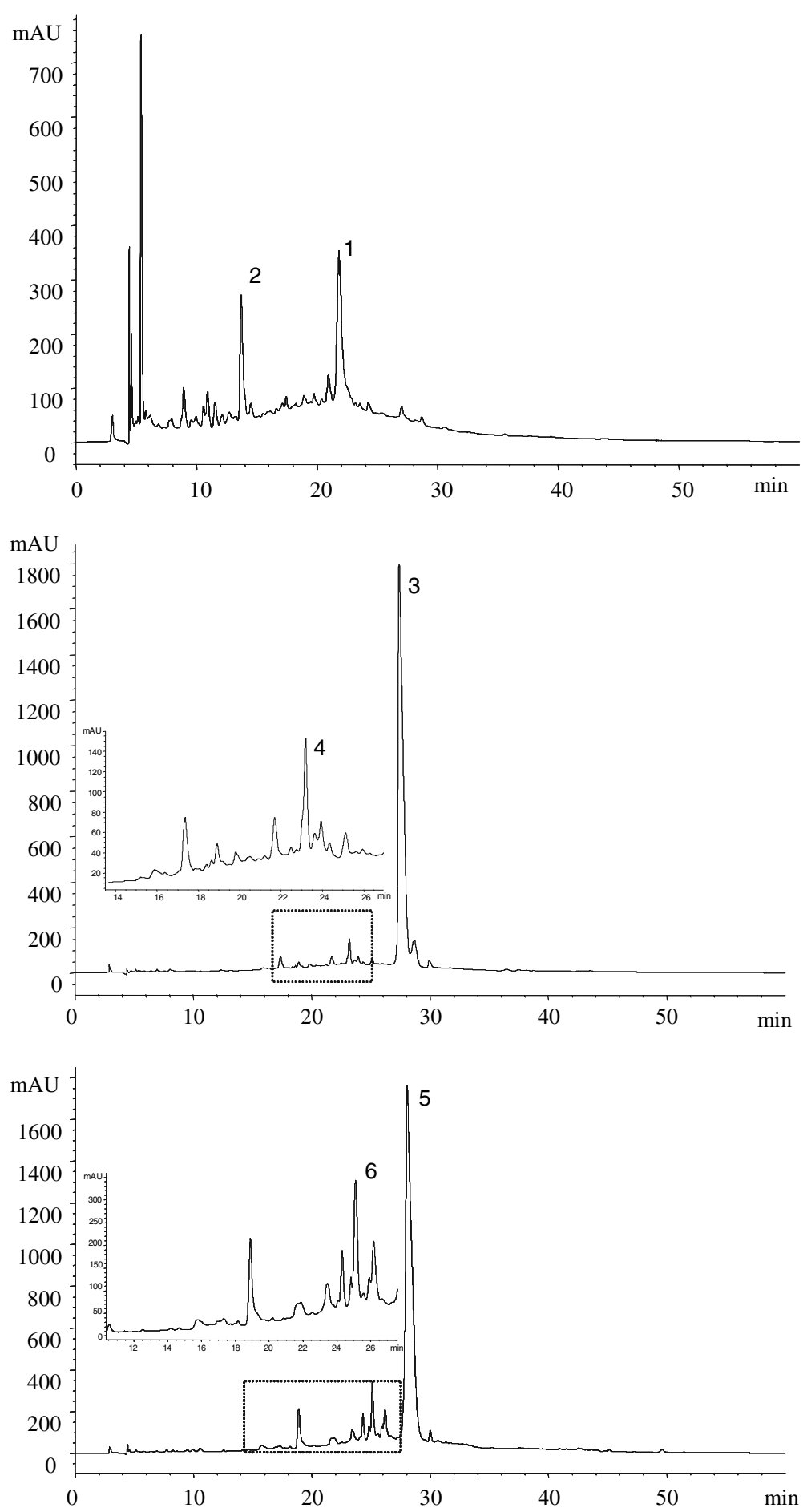

Table 2 Molar concentrations of anthocyanins, phenolic acids from anthocyanin degradation and phenolic acid/anthocyanin molar ratios existing at the end of the study period in the solutions that initially contained $1.2 \mathrm{mmol} / \mathrm{L}$

\begin{tabular}{lllll}
\hline Anthocyanin & $\begin{array}{l}\text { mmol/L of the anthocyanin } \\
\text { (beginning of the study) }\end{array}$ & $\begin{array}{l}\mathrm{mmol} / \mathrm{L} \text { of the anthocyanin } \\
\text { (end of the study) }\end{array}$ & $\begin{array}{l}\text { mmol/L of phenolic } \\
\text { acid formed } \\
\text { (end of the study) }\end{array}$ & $\begin{array}{l}\text { Molar ratio phenolic acid/ } \\
\text { anthocyanin } \\
\text { (end of the study) }\end{array}$ \\
\hline Mv3g & 1.22 & 1.20 & 0.044 & $1: 27$ \\
Pn3g & 1.30 & 1.23 & 0.038 & $1: 30$ \\
Dp3g & 1.29 & 0.44 & 1.13 & $3: 1$ \\
\hline
\end{tabular}


Table 3 Values obtained for $L^{*}, C_{\mathrm{ab}}{ }^{*}$ and $h_{\mathrm{ab}}$ in the solutions of Dp3g, Pn3g and Mv3g corresponding to 1, 7, 14, 21 and 28 days

\begin{tabular}{|c|c|c|c|c|c|c|c|c|c|c|c|}
\hline Dp3g 1 day & $L^{*}$ & $C_{\mathrm{ab}}^{*}$ & $h_{\mathrm{ab}}$ & Pn3g 1 day & $L^{*}$ & $C_{\mathrm{ab}}^{*}$ & $h_{\mathrm{ab}}$ & Mv3g 1 day & $L^{*}$ & $C_{\mathrm{ab}}^{*}$ & $h_{\mathrm{ab}}$ \\
\hline W-0.2 & 93.9 & 14.3 & 355.3 & W-0.2 & 93.2 & 19.4 & 6.1 & $\mathrm{~W}-0.2$ & 90.6 & 17.4 & 351.8 \\
\hline S-0.2 & 87.9 & 15.8 & 354.1 & S- -0.2 & 88.4 & 22.3 & 1.7 & S- -0.2 & 84.7 & 21.2 & 354.9 \\
\hline W-0.4 & 84.0 & 27.3 & 358.9 & W-0.4 & 81.3 & 36.9 & 9.9 & W-0.4 & 78.1 & 31.9 & 356.8 \\
\hline S-0.4 & 82.1 & 31.8 & 356.3 & S-0.4 & 78.2 & 40.8 & 7.8 & S-0.4 & 67.9 & 35.2 & 352.9 \\
\hline W-0.8 & 68.6 & 47.3 & 4.9 & W-0.8 & 67.0 & 58.5 & 18.5 & W-0.8 & 48.3 & 42.0 & 10.7 \\
\hline S-0.8 & 63.7 & 50.8 & 3.2 & S-0.8 & 61.6 & 62.7 & 18.8 & S- -0.8 & 48.8 & 54.0 & 1.4 \\
\hline $\mathrm{W}-1.2$ & 56.9 & 58.7 & 11.2 & W-1.2 & 55.5 & 69.7 & 27.5 & W-1.2 & 50.0 & 60.0 & 10.0 \\
\hline S-1.2 & 50.5 & 60.7 & 10.6 & S-1.2 & 50.1 & 74.5 & 29.1 & S-1.2 & 32.3 & 57.2 & 11.9 \\
\hline Dp3g 14 days & $L^{*}$ & $C_{\mathrm{ab}}^{*}$ & $h_{\mathrm{ab}}$ & Pn3g 14 days & $L^{*}$ & $C_{\mathrm{ab}}^{*}$ & $h_{\mathrm{ab}}$ & Mv3g 14 days & $L^{*}$ & $C_{\mathrm{ab}}^{*}$ & $h_{\mathrm{ab}}$ \\
\hline W-0.2 & 88.9 & 14.4 & 14.0 & $\mathrm{~W}-0.2$ & 85.7 & 23.5 & 15.2 & W-0.2 & 90.6 & 17.4 & 351.8 \\
\hline S- -0.2 & 90.5 & 18.9 & 356.0 & S-0.2 & 85.0 & 30.8 & 3.8 & S- -0.2 & 83.1 & 28.1 & 356.6 \\
\hline W-0.4 & 71.9 & 28.7 & 14.3 & W-0.4 & 76.3 & 40.6 & 19.7 & W-0.4 & 69.4 & 37.5 & 12.3 \\
\hline S- 0.4 & 69.8 & 40.1 & 6.3 & S-0.4 & 71.9 & 51.8 & 14.7 & S- 0.4 & 56.3 & 53.7 & 4.0 \\
\hline W-0.8 & 55.7 & 47.9 & 21.7 & $\mathrm{~W}-0.8$ & 56.4 & 62.9 & 30.1 & W-0.8 & 48.3 & 52.3 & 19.8 \\
\hline S-0.8 & 46.7 & 57.5 & 17.6 & S-0.8 & 48.4 & 77.1 & 33.5 & S-0.8 & 28.1 & 57.8 & 20.8 \\
\hline W-1.2 & 39.0 & 54.5 & 27.6 & W-1.2 & 47.1 & 71.5 & 35.9 & W-1.2 & 26.1 & 53.9 & 29.6 \\
\hline S-1.2 & 26.2 & 56.2 & 28.8 & S-1.2 & 28.2 & 69.1 & 40.0 & S-1.2 & 7.0 & 35.8 & 18.9 \\
\hline Dp3g 28 days & $L^{*}$ & $C_{\mathrm{ab}}^{*}$ & $h_{\mathrm{ab}}$ & Pn3g 28 days & $L^{*}$ & $C_{\mathrm{ab}}^{*}$ & $h_{\mathrm{ab}}$ & Mv3g 28 days & $L^{*}$ & $C_{\mathrm{ab}}^{*}$ & $\overline{h_{\mathrm{ab}}}$ \\
\hline W-0.2 & 83.2 & 14.4 & 14.0 & W-0.2 & 86.8 & 24.9 & 13.6 & W-0.2 & 86.2 & 20.2 & 9.4 \\
\hline S- 0.2 & 81.6 & 19 & 14.3 & S- -0.2 & 80.5 & 32.4 & 16.7 & S-0.2 & 78.0 & 30.1 & 4.7 \\
\hline W-0.4 & 56.2 & 29.6 & 30.5 & W-0.4 & 74.6 & 43.2 & 20.0 & W-0.4 & 68.0 & 40.1 & 16.7 \\
\hline S- 0.4 & 63.1 & 40.5 & 15.9 & S- 0.4 & 64.9 & 56.4 & 23.6 & S-0.4 & 51.5 & 55.9 & 11.4 \\
\hline W-0.8 & 49.7 & 46.6 & 22.4 & W-0.8 & 55.7 & 67.2 & 32.0 & W-0.8 & 50.6 & 60.0 & 25.6 \\
\hline S-0.8 & 39.6 & 52.9 & 22.8 & S-0.8 & 42.5 & 79.4 & 38.3 & S-0.8 & 28.7 & 63.6 & 27.4 \\
\hline W-1.2 & 34.2 & 53.8 & 32.5 & W-1.2 & 38.4 & 69.0 & 38.5 & W-1.2 & 23.7 & 58.7 & 35.1 \\
\hline S-1.2 & 21.7 & 53.9 & 32.6 & S-1.2 & 3.5 & 18.5 & 18.5 & S-1.2 & 7.8 & 38.6 & 20.2 \\
\hline
\end{tabular}

$W$ Values obtained after multiplying the absorbance values of the solutions diluted 20-fold by the dilution factor and $S$ values of the original undiluted solutions. Concentration of the solutions $(\mathrm{mmol} / \mathrm{L})$ is indicated after $\mathrm{W} / \mathrm{S}$

due to the existence of self-association with the increase in anthocyanin concentration should be responsible for the observed descent in the $L^{*}$ values, which indicates that a darkening is produced.

During the storage period ( 28 days) the chroma and lightness differences between the associated and non- associated solutions ( $\Delta C$ and $\Delta L)$ became more pronounced, in consonance with the indicated increase of the extension of the self-association process.

Self-association seems to produce a slight displacement of the angle of hue. In this way, the hue values tend to diminish (more purplish solutions) in the case
Table 4 Color differences $\left(\Delta E_{\mathrm{ab}}^{*}\right)$ obtained in the solutions of Dp3g, Pn3g and Mv3g corresponding to 1,7 , 14,21 and 28 days. The values were obtained taking as reference the values obtained after multiplying by the dilution factor the absorbance values of the same solution diluted 20 -fold

\begin{tabular}{|c|c|c|c|c|c|}
\hline & $\Delta E$ (1 day) & $\Delta E$ (7 days) & $\Delta E$ (14 days) & $\Delta E$ (21 days) & $\Delta E$ (28 days) \\
\hline \multicolumn{6}{|c|}{ Dp3g $(\mathrm{mmo} / \mathrm{L})$} \\
\hline 0.2 & 6.2 & 1.8 & 7 & 7.2 & 7 \\
\hline 0.4 & 5.1 & 6.4 & 12.5 & 15.6 & 15.1 \\
\hline 0.8 & 6.2 & 6.9 & 13.7 & 16 & 15.8 \\
\hline 1.2 & 6.8 & 8.9 & 13.9 & 16.5 & 16 \\
\hline \multicolumn{6}{|c|}{$\operatorname{Pn} 3 g(\mathrm{mmo} / \mathrm{L})$} \\
\hline $0.2^{\circ}$ & 5.8 & 4 & 9.1 & 10 & 9.2 \\
\hline 0.4 & 5.2 & 7.5 & 12.6 & 16.7 & 15.2 \\
\hline 0.8 & 6.9 & 11 & 16.7 & 19.8 & 18.7 \\
\hline 1.2 & 7.4 & 12.3 & 19.7 & 26.6 & 26.2 \\
\hline \multicolumn{6}{|c|}{ Mv3g (mmo/L) } \\
\hline 0.2 & 7.1 & 3.5 & 13.2 & 13 & 11.2 \\
\hline 0.4 & 11 & 10.2 & 21.8 & 23.3 & 22.2 \\
\hline 0.8 & 14.3 & 15.9 & 21 & 22.3 & 20.3 \\
\hline 1.2 & 17.9 & 19.7 & 27.6 & 28.4 & 26.4 \\
\hline
\end{tabular}


of anthocyanins trisubstituted in the B-ring (Mv3g and Dp3g) and to increase (shift towards reddish) in the disubstituted B-ring anthocyanin (Pn3g).

The colour differences $\left(\Delta E_{\mathrm{ab}}^{*}\right)$ attributable to selfassociation were calculated (Table 4). Considering that values of $\Delta E_{\mathrm{ab}}^{*}$ greater than three indicate differences in colour perceptible to the human eye [29], it is concluded that in all the solutions, and usually from their preparation, an evident influence of the self-association on the colour exists. In general, this influence was greater in the solutions of $\mathrm{Mv} 3 \mathrm{~g}$. It was also observed that the values of $\Delta E_{\mathrm{ab}}^{*}$ increased with time. The greatest differences (i.e., maximum effect of self-association) were reached around 14-21 days of storage in the cases of the more concentrated solutions. In the case of the solutions of concentration $<0.2 \mathrm{mmol} / \mathrm{L}$, the process was slower and the maximum differences were reached after 21 days (data not shown). All these observations are coherent with the ones previously made in relation to the above discussed for the process of self-association.

Copigmentation has been demonstrated to occur in red wines [2-5] and indicated to contribute to a great extent $(30-50 \%)$ to the wine colour [15]. The results here obtained suggest that a part of this effect may be due to the existence of self-association between anthocyanins themselves. Studies are currently under way to confirm this supposition and establish the contribution of self-association to copigmentation effects on wine colour.

Acknowledgments Financial support received from INIA (Grant ref. VIN03-043-C3) and CICYT (Grant ref. AGL200200167) is greatly acknowledged. Author M. Dueñas thanks the Spanish Juan de la Cierva program for a grant and Mr. G. H. Jenkins for his help with the English version of the ms.

\section{References}

1. Boulton RBA (1996) Presented at the forty-seventh annual meeting of the American society for enology and viticulture. Reno, NV
2. Asen S, Stewart RN, Norris KH (1972) Phytochemistry 11:1139-1144

3. Brouillard R, Wigand MC, Dangles O, Cheminat A (1991) J Chem Soc Perkin Trans 2:1235-1241

4. Liao H, Cai Y, Haslam E (1992) J Sci Food Agric 59:299-305

5. Davies AJ, Mazza G (1993) J Agric Food Chem 41:716-720

6. Goto T, Kondo T (1991) Angew Chem Int Ed Engl 30:17-33

7. Hoshino T, Matsumoto U, Goto T (1980) Phytochemistry 19:663-667

8. Hoshino T, Matsumoto U, Goto T (1981) Phytochemistry 20:1971-1976

9. Hoshino T, Matsumoto U, Harada N, Goto T (1981) Tetrahedron Lett 22:3621-3624

10. Hoshino T, Matsumoto U, Goto T, Harada N (1982) Tetrahedron Lett 23:433-436

11. Hoshino T, Matsumoto U (1980) Tetrahedron Lett 21:17511754

12. Timberlake CF (1980) Food Chem 5:69-80

13. Miniati E, Damiani P, Mazza G (1992) Ital J Food Sci 4:109_ 116

14. Houbiers C, Lima JC, Maçanita AL, Santos H (1998) J Phys Chem B 102:3578-3585

15. Boulton R (2001) Am J Enol Vitic 52:67-84

16. Hoshino T (1991) Phytochemistry 30:2049-2055

17. Somers TC, Evans ME (1979) J Sci Food Agric 30:623-633

18. Heredia FJ, Francia-Aricha EM, Rivas-Gonzalo JC, Vicario IM, Santos Buelga C (1998) Food Chem 63:491-498

19. Dueñas M, Salas E, Cheynier V, Dangles O, Fulcrand H (2006) J Agric Food Chem 54:189-196

20. Dangles O, Saito N, Brouillard R (1993) J Am Chem Soc 115:3125-3132

21. Alcalde-Eon C, Escribano-Bailón MT, Santos-Buelga C, Rivas-Gonzalo J (2004) Anal Chim Acta 513:305-318

22. Heredia FJ, Álvarez C, González-Miret ML, Ramírez A (2004) Registro General de la Propiedad Intelectual SE1052-04 Sevilla, Spain

23. CIE 15:2004, Technical report colorimetry, 3rd edn. CIE Central Bureau, 2004. ISBN 3901906339

24. Figueiredo P, Elhabiri M, Toki K, Saito N, Dangles O, Brouillard R (1995) Phytochemistry 41:301-308

25. Markakis P (1982) Stability of anthocyanins in foods. In: Markakis P (ed) Anthocyanins as food colors, 1st edn. Academic, New York, 163-178

26. Furtado P, Figueiredo P, Chaves-das-Neves H, Pina FJ (1993) Photochem Photobiol A 78:113-118

27. Eiro MJ, Heinonen M (2002) J Agric Food Chem 50:74617466

28. Nuñez V, Monagas M, Gómez-Cordovés MC, Bartolomé B (2004) Postharvest Biol Technol 31:69-79

29. Martínez JA, Melgosa M, Pérez M, Hita E, Negueruela AI (2001) Food Sci Technol Int 7 5:439-444 